\title{
Hybrid organic-inorganic inks flatten the energy landscape in colloidal quantum dot solids
}

\begin{abstract}
Mengxia Liu, Oleksandr Voznyy, Randy Sabatini, F. Pelayo García de Arquer, Rahim Munir, Ahmed Hesham Balawi, Xinzheng Lan, Fengjia Fan, Grant Walters, Ahmad R. Kirmani, Sjoerd Hoogland, Frédéric Laquai, Aram Amassian, and Edward H. Sargent
\end{abstract}

Version Post-Print/Accepted Manuscript

Citation Liu, M., Voznyy, O., Sabatini, R., García de Arquer, F., Munir, R., \& (published version) Balawi, A. et al. (2016). Hybrid organic-inorganic inks flatten the energy landscape in colloidal quantum dot solids. Nature Materials. http://dx.doi.org/10.1038/nmat4800

Publisher's Statement The final published version is available at Nature Publishing Group via http://dx.doi.org/10.1038/nmat4800.

Always cite the published version, so the author(s) will receive recognition through services that track citation counts, e.g. Scopus. If you need to cite the page number of the TSpace version (original manuscript or accepted manuscript) because you cannot access the published version, then cite the TSpace version in addition to the published version using the permanent URI (handle) found on the record page. 


\section{Hybrid Organic-Inorganic Inks Flatten the Energy Landscape in Colloidal Quantum Dot Solids}

Mengxia Liu, ${ }^{1}$ Oleksandr Voznyy, ${ }^{1}$ Randy Sabatini, ${ }^{1}$ F. Pelayo García de Arquer, ${ }^{1}$ Rahim Munir, ${ }^{2}$ Ahmed Hesham Balawi, ${ }^{2}$ Xinzheng Lan, ${ }^{1}$ Fengjia

Fan, ${ }^{1}$ Grant Walters, ${ }^{1}$ Ahmad R. Kirmani, ${ }^{2}$ Sjoerd Hoogland, ${ }^{1}$ Frédéric Laquai, ${ }^{2}$ Aram Amassian, ${ }^{2}$ Edward H. Sargent ${ }^{1 *}$

${ }^{1}$ Department of Electrical and Computer Engineering, University of Toronto, 10 King’s College Road, Toronto, Ontario, M5S 3G4, Canada

${ }^{2}$ King Abdullah University of Science and Technology (KAUST), KAUST Solar Center (KSC), and Physical Sciences and Engineering Division, 4700

KAUST, Thuwal 23955-6900, Saudi Arabia

*E-mail: ted.sargent@ utoronto.ca (E.H.S.) 
Bandtail states in disordered semiconductor materials result to losses in open-circuit voltage $\left(V_{o c}\right)$ and inhibit carrier transport in photovoltaics. For colloidal quantum dot (CQD) films that promise low-cost, large-area, air-stable photovoltaics, bandtails are determined by CQD synthetic polydispersity and inhomogeneous aggregation during the ligand exchange process. Here we introduce a new method for the synthesis of solution-phase ligand-exchanged CQD inks that enable a flat energy landscape and an advantageously high packing density. In the solid-state, these materials exhibit a sharper bandtail and reduced energy funneling compared to the previous best CQD thin films for photovoltaics. Consequently, we demonstrate solar cells with higher $V_{\text {oc }}$ and more efficient charge injection into the electron acceptor, allowing the use of a closer-to-optimum bandgap to absorb more light. These enable the fabrication of CQD solar cells made via a solution-phase ligand-exchange, with a certified power conversion efficiency of $11.28 \%$. The devices are stable when stored in air, unencapsulated, for over 1000 hours. 
Colloidal quantum dots (CQDs), prized for their size-tunable bandgap, solution processability and high monodispersity ${ }^{1-4}$, have generated great interest for their applications in optoelectronic devices including photodetectors ${ }^{5,6}$, light-emitting diodes ${ }^{7}$, lasers $^{8}$, and solar cells ${ }^{9,10}$. Rapid progress in surface passivation ${ }^{10-13}$ and device architecture $^{9,14}$ has propelled the certified power conversion efficiency (PCE) of CQD solar cells to $10.6 \%{ }^{15}$. Significant device stability has also been achieved, with devices exhibiting no loss in PCE over 150 days in air ambient ${ }^{9}$. In addition to their promise in single-junction devices, CQD solar cells are attractive as back cells that utilize unharvested infrared light transmitted through silicon and perovskites ${ }^{16}$.

The PCE of CQD solar cells has been steadily growing over time as research efforts addressed the passivation and transport properties of CQD films with the aid of new ligands ${ }^{17,18}$. One of the remaining problems is the inhomogeneous energy landscape present in CQD solids ${ }^{19-21}$. This arises due to poor monodispersity ${ }^{22}$, random packing ${ }^{23}$, and heterogeneous aggregation of CQDs. An inhomogeneous energy landscape widens the distribution of electronic states at the bandedges, creating bandtail states in both the conduction and valence bands ${ }^{24,25}$. In the case of coupled CQD solids, photoexcited carriers thermalize to the localized tail states in the picosecond regime $e^{26,27}$, leading to losses in opencircuit voltage $\left(\mathrm{V}_{\mathrm{oc}}\right)^{22,28}$, and a rough energy landscape that inhibits carrier transport ${ }^{29}$ and causes band misalignment with electron acceptors.

It is well established that site energy disorder is introduced by CQD size polydispersity ${ }^{30}$. However, another critical source of energetic inhomogeneity, induced by solid-state ligand exchange procedure ${ }^{24}$ used to render CQD films conductive, has been so far largely overlooked. Poor control over this process leads to inconsistent ligand exchanges, 
heterogeneous CQD fusion, and organic residues in the solid-state. The solution-based ligand-exchange method has therefore attracted much attention recently since it has the potential to overcome the above issues; however, it has not yet outperformed ${ }^{19}$ conventional layer-by-layer processing. In particular, stable CQD inks based on organic halide salts, halometallates $\left(\left[\mathrm{PbI}_{3}\right]^{-}\right)$and pseudohalides (thiocyanates, azides) $)^{31-33}$ have been demonstrated. However, the counter-ions necessary for colloidal stabilization in polar solvents cannot be completely removed from the films, and this has been shown to inhibit carrier transport ${ }^{18,34}$. A post-annealing step is thus necessary in order to remove the counterions; though to maintain the charge balance, the surface-bound halides are also unavoidably removed $^{34}$. This leads to a deterioration of surface passivation and a non-uniform aggregation of CQDs. A procedure for removing the unwanted cations prior to film deposition is thus urgently required.

Here we designed a ligand exchange process based on lead halide as a precursor with sub-molar amounts of ammonium acetate assisting the colloidal stabilization $\left(\mathrm{PbX}_{2} / \mathrm{AA}\right.$ exchange). This hybrid CQD ink maximizes the amount of halides on the surface and enables the removal of the original long organic molecules (oleic acid) from the solution. It thereby allows deposition of an active layer that does not require a disruptive placeexchange. By comparing the new ink with the best performing devices fabricated by solidstate layer-by-layer (LBL) and conventional solution exchange methods, we demonstrate the multiple benefits of the flat-energy-landscape films: reduced bandtailing improves $\mathrm{V}_{\mathrm{oc}}$, carrier transport, and charge injection into electron acceptor, allowing for a more optimal CQD bandgap and enhanced light absorption. The higher halide content also provides better 
passivation, enabling thicker active layer devices that absorb a greater proportion of incident solar irradiation.

A schematic of the solution exchange process is presented in Fig. 1. Before the exchange, PbS CQDs are capped with oleic acid and dispersed in octane. During the exchange, the bulky oleic acid ligands are replaced by $\left[\mathrm{PbX}_{3}\right]^{-}$anions with the aid of ammonium protons ${ }^{35}$. Both $\left[\mathrm{NH}_{4}\right]^{+}$and $[\mathrm{PbX}]^{+}$contribute to nanocrystal colloidal stabilization in the polar solvent. After the ligand exchange, CQDs are precipitated via the addition of an anti-solvent. The mixed solvent takes away $\mathrm{NH}_{4} \mathrm{Ac}$ and excess lead halide salts, thus leaving clean $\left[\mathrm{PbX}_{3}\right]^{-} /[\mathrm{PbX}]^{+}$-capped CQD solids without any organic residues. This is markedly different from conventional methylammonium iodide (MAI) ${ }^{34}$ and methylammonium lead iodide $\left(\mathrm{MAPbI}_{3}\right)^{18}$ exchanges, in which the organic cations cannot be removed from the CQD surface without annealing.

X-ray diffraction (Fig. S1) shows the expected PbS crystal peaks in the resultant films ${ }^{36}$. The absorbance spectrum of the CQD solution after exchange preserves the width of the excitonic feature of the original CQDs (Fig. S2). CQD size statistics are obtained from transmission electron microscopy images (Fig. S3). These indicate no change in average size and size distribution following the ligand exchange. The absence of a nitrogen peak from X-ray photoelectron spectroscopy (XPS) of the film (Fig. S4) indicates complete removal of $\left[\mathrm{NH}_{4}\right]^{+}$from the CQD surface. With XPS we further observe a ratio of iodine to lead and bromine to lead of 0.74 and 0.12 respectively. This is significantly higher than previously reported layer-by-layer exchanges employing tetrabutylammonium iodide (TBAI) as a ligand ${ }^{10}$. This indicates nearly full halide coverage of the quantum dot surface, 
which was enabled by augmenting the amount of $[\mathrm{PbX}]^{+}$counterions. It is expected to prevent dot fusion in films and provide higher-quality passivation.

To elucidate the packing density and uniformity of the films, grazing-incidence smallangle X-ray scattering (GISAXS) measurements were carried out. For the $\mathrm{PbX}_{2} / \mathrm{AA}$ exchanged film, intensity accumulates at locations forming a hexagonal pattern, revealing the orientational in-plane ordering of $\mathrm{CQDs}^{37}$ (Fig. 2a). Azimuthal integration of the diffraction pattern, with the high-intensity lobes subtracted from the integration (Fig. 2b), shows an average inter-dot spacing of $3.21 \mathrm{~nm}$ for the $\mathrm{PbX}_{2} / \mathrm{AA}$ exchanged film. Compared to $3.51 \mathrm{~nm}$ for the TBAI solid-state exchanged film, the smaller number reveals that the solution exchange increases the CQD packing density. Notably, it also shows a narrower distribution of the inter-dot spacing for $\mathrm{PbX}_{2} / \mathrm{AA}$ exchange, suggesting more uniform $\mathrm{CQD}$ packing than the controls (Fig. 2b, S5). The enhanced spatial uniformity leads to greater energetic homogeneity and a narrower distribution of the density of states ${ }^{23,38}$, reducing the hopping transport barrier. It is confirmed by UV-Vis absorption (Fig. 2c) that the $\mathrm{PbX}_{2} / \mathrm{AA}$ exchanged film exhibits the narrowest exciton peak with a full-width at half maximum (FWHM) of $156 \mathrm{meV}$ (Table S1). In the case of control samples, calculation taking into account the PbS bandgap dependence on CQD size ${ }^{30}$ and single-dot linewidth ${ }^{39}$ shows that about $50 \%$ of polydispersity is produced by ligand exchange process, making it as important as synthetic control. The avoidance of inhomogeneous CQD aggregation, exemplified by a narrower absorption peak and improved spatial uniformity of $\mathrm{PbX}_{2} / \mathrm{AA}$ exchanged films observed with GISAXS, is expected to contribute to a flatter energy landscape.

To verify the degree of energetic disorder in different films, we studied optical absorption using photothermal deflection spectroscopy (PDS). This technique provides a 
sensitive way to probe tail states below the bandgap ${ }^{40}$ and therefore of estimating the Urbach energy that describes the localized tail states ${ }^{38}$. The sharpest bandtail is detected for $\mathrm{PbX}_{2} / \mathrm{AA}$ exchanged films, which exhibits the lowest Urbach energy of $29 \mathrm{meV}$. This is over $10 \%$ less than TBAI (33 meV) and $30 \%$ less than $\mathrm{MAPbI}_{3}$ exchanged (44 meV) films (Fig. 2d).

To investigate the effect of energetic disorder on energy funneling upon light absorption, we studied the spectral distribution of photoexcited carriers in CQD films using transient absorption (TA) spectroscopy. An excitation density of $\left\langle\mathrm{N}_{\mathrm{abs}}\right\rangle=0.004$ is used to minimize Auger recombination and maintain signal-to-noise ratio (Fig. S6). $\left\langle\mathrm{N}_{\mathrm{abs}}\right\rangle$ is the average number of photons absorbed per CQD, obtained from the product of per-pulse fluence and the absorption cross-section taken from ref. 41. Spectro-temporal TA maps of three different samples are shown in Fig. 3a-c. The positions of photobleach peaks at 1 ps, the time when bleach signal arises, are in good agreement with the exciton peaks from steady-state absorption (Fig. S7). After absorption, the photoexcited carriers quickly funnel to the lowest energy sites, as observed by a red shift of the transient bleach peak (Fig. S8). An $\sim 11$ meV shift is observed for the $\mathrm{PbX}_{2} / \mathrm{AA}$ exchanged film, whereas the shifts for control films are $18 \mathrm{meV}$ for the TBAI solid-state exchange and $28 \mathrm{meV}$ for the $\mathrm{MAPbI}_{3}$ solution exchange (Fig. 3d). The same trend is observed from TA studies of CQD films with various bandgaps and thicknesses (Fig. S9). This trend, consistent with the absorption exciton peak broadening in the exchanged films, correlates to a higher degree of disorder with energy funneling towards undesired bandtail states. The reduced energy funneling in the $\mathrm{PbX}_{2} / \mathrm{AA}$-exchanged film evidences a flatter energy landscape and reduced tail states below the bandgap, suggesting promise for a photovoltaic cell with minimized $\mathrm{V}_{\text {oc }}$ deficit. 
In CQD solar cells, excitons are produced upon solar irradiation and are then quickly transported to the lowest energy states within the CQD film (Fig. 4a). The tail states filled with carriers represent the effective bandgap $\left(\mathrm{E}_{\mathrm{g}}{ }^{\prime}\right)$ that determines the maximum attainable

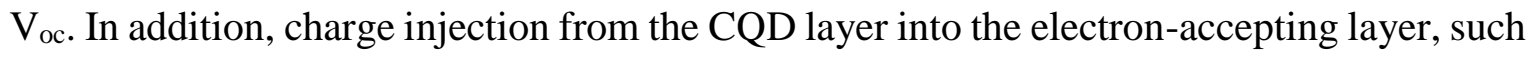
as $\mathrm{ZnO}$, is inhibited by a large number of tail states that are much deeper than the conduction band of $\mathrm{ZnO}$ (Fig. 4a, shaded region). As a consequence, the sharper bandedges (Fig. 4a, solid black line) enable a higher $\mathrm{E}_{\mathrm{g}}$ that leads to a reduced $\mathrm{V}_{\text {oc }}$ deficit, better charge transport, and efficient charge extraction from smaller-bandgap CQDs, more optimal in terms of the amount of absorbed light. This effect has been previously investigated ${ }^{21}$, showing that increased concentration and depth of tail states can deteriorate both voltage and current.

We therefore pursued the realization of enhanced-performance CQD solar cells. The devices are fabricated by depositing the following layers on an ITO electrode: a ZnO electron accepting layer, a thick layer of pre-exchanged PbS CQDs, two thin CQD films treated by 1,2-ethanedithiol (EDT), and Au as the top contact (Fig. S10). Cross-sectional scanning electron microscopy image indicates the presence of two layers, a thick CQD absorber and a thin PbS-EDT layer (Fig. S11).

To confirm and study the flatter energy landscape at a solar cell level, we measured the high dynamic range external quantum efficiency (HDR EQE). With high sensitivity, this technique allows screening the carrier density within bandtails. In good agreement with the material and photophysical characterization, the bandtail EQE of $\mathrm{PbX}_{2} / \mathrm{AA}$-exchanged solar cell is the steepest relative to previous best-performing controls, indicating the sharpest band edge preserved in the final device (Fig. 4b). The obtained sharpening of bandtails can therefore contribute to a major improvement on solar cell performance. 
The device performance was measured under AM1.5 solar illumination. As expected, a higher $V_{o c}$ is achieved when the dots of the same size are used for active layer (Fig. 4c). We further tested the effect of changing the bandgap of PbS CQDs on the performance of devices prepared using different ligand exchange methods. Relatively thin devices are used in order to compare fairly the effect of bandtailing, without penalizing them for difference in carrier diffusion length. $\mathrm{PbX} / \mathrm{AA}$-exchanged films have a consistently higher $\mathrm{V}_{\mathrm{oc}}$, by 25$50 \mathrm{meV}$ compared to control devices. Better band alignment of the CQD bandedge with the $\mathrm{ZnO}$ electrode also allows achieving higher current densities (Fig. 4d). In the case of the control devices, deep bandtails inhibit the electron injection at the interface. As a result, for control devices the optimum performance is typically achieved at a bandgap of $1.43 \mathrm{eV}$, whereas the new solution exchange enables a red-shift of the bandgap towards a more optimal $1.32 \mathrm{eV}$ to maximize the product of $\mathrm{V}_{\mathrm{oc}}$ and short-circuit current ( $\mathrm{J}_{\mathrm{sc}}$ ) (Fig. $\mathrm{S} 12$ ).

To study the improvements enabled by better surface passivation and carrier mobility (Fig. S13) as a result of increased halide surface coverage, we investigated the thicknessdependent $\mathrm{PCE}$ for our best $\mathrm{PbX}_{2} / \mathrm{AA}$-exchanged and control devices. Optimum device thickness is determined by the carrier diffusion length, which in turn depends on the trap density, requiring device thickness to not exceed the sum of diffusion and drift lengths ${ }^{42,43}$. Beyond this thickness, trap recombination losses increase, deteriorating all device parameters: $\mathrm{V}_{\mathrm{oc}}, \mathrm{J}_{\mathrm{sc}}$, and fill factor (FF). Strikingly, for the $\mathrm{PbX} / \mathrm{AA}$ exchanged device, the $\mathrm{J}_{\mathrm{sc}}$, as well as PCE, improves as the film thickness increases, without sacrificing $\mathrm{V}_{\mathrm{oc}}$ and FF (Fig. 4e, Fig. S14). This suggests better passivation and an enhanced diffusion length. The best performing device with a $350 \mathrm{~nm} \mathrm{PbX}_{2} / \mathrm{AA}$-exchanged $\mathrm{CQD}$ absorber layer demonstrated a certified AM1.5 PCE of 11.28\% (Fig. 5a,b and Fig. S15). The device 
achieved a $J_{\mathrm{sc}}$ of $27.23 \mathrm{~mA} \mathrm{~cm}$ and a $\mathrm{V}_{\text {oc }}$ of $0.61 \mathrm{~V}$ with a CQD bandgap in solution of $1.32 \mathrm{eV}$. These devices exhibit reproducibly high efficiencies (Fig. S16). They retain around 90\% of their initial PCE after $1000 \mathrm{~h}$ of storage in air, unencapsulated (Fig. 5c).

In summary, we have introduced a new solution-phase ligand-exchange method that enables closely packed CQD films with flat energy landscapes. The lead halide precursors with addition of ammonium acetate allow efficient ligand exchange and provide improved passivation of CQD solids. Organic residues are removed via nanocrystal precipitation, and as a result, clean CQD films can be deposited without any post-treatment. Decreased CQD aggregation and subsequent bandtailing reduces energy funneling, improves carrier transport, and facilitates efficient carrier injection into electrodes. With these benefits, we were able to increase the device thickness and reduce the absorber bandgap to gain more current. Record certified performance quantum dot solar cells have been achieved, with a $350 \mathrm{~nm}$ solution-exchanged active layer. The present work suggests that, with further progress, the benefits of colloidal quantum dots - in size-tunable bandgap for full-spectra current absorption, as well as accelerated lifetime under room atmosphere - can be developed into high efficiency solar cells via sharpening of bandtails.

\section{Acknowledgements}

This publication is based in part on work supported by Award KUS-11-009-21, made by King Abdullah University of Science and Technology (KAUST), by the Ontario Research Fund Research Excellence Program, and by the Natural Sciences and Engineering Research Council (NSERC) of Canada. F. P. García de Arquer acknowledges financial support from the Connaught fund. A.H.B and F.L. thank Koen Vandewal for his 
contribution to the PDS setup and Markus Baier for help with the experiments. The authors

thank E. Palmiano, L. Levina, R. Wolowiec, D. Kopilovic, G. Kim and F. Fan for their help during the course of study.

\section{References}

(1) McDonald, S. A. et al. Solution-processed $\mathrm{PbS}$ quantum dot infrared photodetectors and photovoltaics. Nat. Mater. 4, 138-142 (2005).

(2) Kamat, P. V. Quantum dot solar cells. Semiconductor nanocrystals as light harvesters. J. Phys. Chem. C. 112, 18737-18753 (2008).

(3) Luther, J. M. et al. Schottky solar cells based on colloidal nanocrystal films. Nano Lett. 8, 3488-3492 (2008).

(4) Nozik, A. J. et al. Semiconductor quantum dots and quantum dot arrays and applications of multiple exciton generation to third-generation photovoltaic solar cells. Chem. Rev. 110, 6873-6890 (2010).

(5) Konstantatos, G. et al. Ultrasensitive solution-cast quantum dot photodetectors. Nature. 442, 180-183 (2006).

(6) Lee, J.-S., Kovalenko, M. V., Huang, J., Chung, D. S. \& Talapin, D. V. Band-like transport, high electron mobility and high photoconductivity in all-inorganic nanocrystal arrays. Nat. Nanotechnol. 6, 348-352 (2011).

(7) Sun, Q. J. et al. Bright, multicoloured light-emitting diodes based on quantum dots. Nat. Photonics. 1, 717-722 (2007).

(8) Hoogland, S. et al. A Solution-processed 1.53 um quantum dot laser with temperatureInvariant emission wavelength. Opt. Express. 14, 3273-3281 (2006).

(9) Chuang, C.-H. M., Brown, P. R., Bulović, V. \& Bawendi, M. G. Improved performance and stability in quantum dot solar cells through band alignment engineering. Nat. Mater. 13, 796-801 (2014).

(10) Lan, X. et al. Passivation using molecular halides increases quantum dot solar cell performance. Adv. Mater. 28, 299-304 (2016).

(11) Ip, A. H. et al. Hybrid passivated colloidal quantum dot solids. Nat. Nanotechnol. 7, 577-582 (2012).

(12) Tang, J. et al. Colloidal-quantum-dot photovoltaics using atomic-ligand passivation. Nat. Mater. 10, 765-771 (2011).

(13) Ning, Z. et al. All-inorganic colloidal quantum dot photovoltaics employing solutionphase halide passivation. Adv. Mater. 24, 6295-6299 (2012).

(14) Ning, Z. et al. Air-stable n-type colloidal quantum dot solids. Nat. Mater. 13, 822-828 (2014).

(15) NREL chart. http://www.nrel.gov/ncpv/images/efficiency_chart.jpg

(16) Ip, A. H. et al. Infrared colloidal quantum dot photovoltaics via coupling enhancement and agglomeration suppression. ACS Nano. 9, 8833-8842 (2015).

(17) Carey, G. H., Levina, L., Comin, R., Voznyy, O. \& Sargent, E. H. Record charge carrier diffusion length in colloidal quantum dot solids via mutual dot-To-dot surface passivation. Adv. Mater. 27, 3325-3230 (2015). 
(18) Yang, Z. et al. Colloidal quantum dot photovoltaics enhanced by perovskite shelling. Nano Lett. 15, 7539-7543 (2015).

(19) Pejova, B. \& Abay, B. Nanostructured CdSe films in low size-quantization regime: temperature dependence of the band gap energy and sub-band gap absorption tails. $J$. Phys. Chem. C. 115, 23241-23255 (2011).

(20) Pejova, B., Abay, B. \& Bineva, I. Temperature dependence of the band-gap energy and sub-band-gap absorption tails in strongly quantized $\mathrm{ZnSe}$ nanocrystals deposited as thin films. J. Phys. Chem. C. 114, 15280-15291 (2010).

(21) Zhitomirsky, D. et al. Colloidal quantum dot photovoltaics: The effect of polydispersity. Nano Lett. 12, 1007-1012 (2012).

(22) Guyot-Sionnest, P. Electrical transport in colloidal quantum dot films. J. Phys. Chem. Lett. 3, 1169-1175 (2012).

(23) Sa-Yakanit, V. \& Glyde, H. R. Urbach tails and disorder. Comments Cond. Mat. Phys. 13, 35 (1987).

(24) Erslev, P. T. et al. Sharp exponential band tails in highly disordered lead sulfide quantum dot arrays. Phys. Rev. B. 86, 155313-155316 (2012).

(25) Kagan, C. R. \& Murray, C. B. Charge transport in strongly coupled quantum dot solids. Nature Nanotechnology 10, 1013-1026 (2015)

(26) Hess, K., Leburton, J. P. \& Ravaioli, U. Hot Carriers in Semiconductors Ch. 3 (1996 Plenum Press, New York, 1996).

(27) Gao, Y. et al. Enhanced Hot-Carrier Cooling and Ultrafast Spectral Diffusion in Strongly Coupled PbSe Quantum-Dot Solids. Nano Lett. 11, 5471-5476 (2011).

(28) Chuang, C.-H. M. et al. Open-circuit voltage deficit, radiative sub-bandgap states, and prospects in quantum dot solar cells. Nano Lett. 15, 3286-3294 (2015).

(29) Gao, J. \& Johnson, J. C. Charge trapping in bright and dark states of coupled PbS quantum dot films. ACS Nano. 6, 3292-3303 (2012).

(30) Weidman, M. C., Beck, M. E., Hoffman, R. S., Prins, F. \& Tisdale, W. A. Monodisperse, air-stable $\mathrm{PbS}$ nanocrystals via precursor stoichiometry control. ACS Nano. 8, 63636371 (2014).

(31) Zhang, H., Jang, J., Liu, W. \& Talapin, D. V. Colloidal nanocrystals with inorganic halide, pseudohalide, and halometallate ligands. ACS Nano, 8, 7359-7369 (2014).

(32) Nag, A., Zhang, H., Janke, E. \& Talapin, D. V. Inorganic surface ligands for colloidal nanomaterials. Z. Phys. Chem. 229, 85-107 (2015).

(33) Dirin, D. N. et al. Lead halide perovskites and other metal halide complexes as inorganic capping ligands for colloidal nanocrystals. J. Am. Chem. Soc. 136, 6550-6553 (2014).

(34) Ning, Z., Dong, H., Zhang, Q., Voznyy, O \& Sargent, E. H. Solar cells based on inks of n-Type colloidal quantum dots. ACS Nano. 8, 10321-10327 (2014).

(35) Balazs, D. M. et al. Counterion-mediated ligand exchange for PbS colloidal quantum dot superlattices. ACS Nano. 9, 11951-11959 (2015).

(36) Tang, J. et al. Quantum dot photovoltaics in the extreme quantum confinement regime: The surface-chemical origins of exceptional air-and light-stability. ACS Nano 4, 869878 (2010).

(37) Bian, K. et al. Shape-anisotropy driven symmetry transformations in nanocrystal superlattice polymorphs. ACS Nano. 5, 2815-2823 (2011).

(38) John, S. Theory of electron band tails and Urbach optical-absorption edge. Pyhs. Rev. Lett. 57, 1777-1780 (1986). 
(39) Peterson, J. J. \& Krauss, T. D. Fluorescence spectroscopy of single lead sulfide quantum dots. Nano Lett. 6, 510-514 (2006).

(40) Venkateshvaran, D. et al. Approaching disorder-free transport in high-mobility conjugated polymers. Nature 515, 384-388 (2014).

(41) Moreels, I. et al. Size-dependent optical properties of colloidal PbS quantum dots. ACS Nano. 3, 3023-3030 (2009).

(42) Pattantyus-Abraham, A. G. et al. Depleted-heterojunction colloidal quantum dot solar cells. ACS Nano. 4, 3374-3380 (2010).

(43) Zhitomirsky, D., Voznyy, O., Hoogland, S. \& Sargent, E. H. Measuring Charge Carrier Diffusion in Coupled Colloidal Quantum Dot Solids. ACS Nano. 7, 5282-5290 (2013). 


\section{Methods}

1) Ligand Exchange and Film Fabrication. Oleic-acid-capped CQDs were synthesized using previously published methods ${ }^{44}$. $\mathrm{PbX}_{2} / \mathrm{AA}$ solution-phase ligand-exchange process was carried out in a test tube in air. Lead halides (lead iodide $0.1 \mathrm{M}$ and lead bromide $0.02 \mathrm{M}$ ) and $\mathrm{NH}_{4} \mathrm{Ac}(0.04 \mathrm{M})$ are pre-dissolved in dimethylformamide (DMF). A $5 \mathrm{~mL}$ amount of CQD octane solution $(10 \mathrm{mg} / \mathrm{mL})$ was added to a $5 \mathrm{~mL}$ of precursor solution. These were mixed vigorously for 1-2 min until the CQDs completely transferred to the DMF phase. The DMF solution was washed three times with octane. After ligand exchange, CQDs were precipitated via the addition of toluene, and were separated by centrifugation. After 20 min of drying, the CQDs were then redispersed in butylamine $(200 \mathrm{mg} / \mathrm{mL})$ to facilitate the film deposition. The exchanged ink was deposited by single-step spin-coating at $2500 \mathrm{rpm}$ for $30 \mathrm{~s}$ to achieve $\sim 350 \mathrm{~nm}$ thickness. The TBAI solid-state exchanged and $\mathrm{MAPbI}_{3}$ solutionphase exchanged films were fabricated following previously published recipes ${ }^{10,18}$.

2) CQD Solar Cell Fabrication. The $\mathrm{ZnO}$ nanoparticles were synthesized following a published method ${ }^{9}$. The $\mathrm{ZnO}$ solution was spin-cast onto ITO glass at $3000 \mathrm{rpm}$ for $20 \mathrm{~s}$. The first layer of $\mathrm{PbS}$ CQDs was deposited onto $\mathrm{ZnO}$ substrate using the $\mathrm{PbX}_{2} / \mathrm{AA}$ exchanged ink. Then, two PbS-EDT layers were fabricated via a layer-by-layer method. Oleic-acid-capped CQDs were deposited, and then a 0.01 vol\% EDT in acetonitrile solution was applied to the film for $30 \mathrm{~s}$, followed by a 3-time acetonitrile rinsing step. On top of the PbS CQD films, $120 \mathrm{~nm}$ Au was deposited as the top electrode. The solar cell area is 0.049 $\mathrm{cm}^{2}$. 


\section{3) Grazing Incidence Small-Angle X-ray Spectroscopy (GISAXS). GISAXS was}

performed at the D1 beamline, Cornell High Energy Synchrotron Source (CHESS). Wavelength of the employed X-ray beam was $1.155 \AA$. A wide band pass (1.47\%) doublebounce multilayer monochromator was used. The scattering patterns were obtained at a photon-incident angle of 0.5 degrees with respect to the sample plane.

4) X-ray Photoelectron Spectroscopy (XPS) Measurements. XPS measurements were carried out using a Thermo Scientific K-Alpha system, with a $75 \mathrm{eV}$ pass energy, and binding energy steps of $0.05 \mathrm{eV}$. All signals are normalized to $\mathrm{Pb}$.

5) Transient Absorption Measurements. Femtosecond laser pulses were generated by a regeneratively amplified $\mathrm{Yb}: \mathrm{KGW}$ laser at a $5 \mathrm{kHz}$ repetition rate (Light Conversion, Pharos). The $1030 \mathrm{~nm}$ fundamental passes through a beam splitter, where most of the beam is sent into an optical parametric amplifier (Light Conversion, Orpheus) to generate $400 \mathrm{~nm}$, $\sim 350$ fs duration pulses for the pump. The rest of the fundamental is focused into a near-IR continuum generation crystal (Ultrafast, Helios), translated at $1 \mathrm{~mm} / \mathrm{s}$, to generate the whitelight continuum. The pump pulse and probe were sent into an optical bench (Ultrafast, Helios). The time delay was adjusted by changing the pathlength of the probe, up to a delay of 8 ns. An optical chopper was used to block every other pump pulse. The signal was collected into a CCD camera after dispersion by a grating spectrograph (Ultrafast, Helios). Kinetic traces were fitted to the convolution of the instrument response and a sum of exponential decays. Time zero was allowed to vary with wavelength to account for the chirp of the probe. All TA measurement results were plotted based on the ref ${ }^{45}$. 
6) Photo-thermal Deflection Spectroscopy (PDS) Measurements. PDS was performed using a custom PDS setup. Light from a $250 \mathrm{~W}$ quartz-tungsten-halogen lamp (Newport 66996-250Q-R1) was sent through a monochromator (LOT MSH-300) and used as pump allowing excitation across the UV to NIR spectral region. The pump light was modulated by a chopper operating at a constant frequency of few Hertz and focused on the sample, which itself was immersed in a chemically inert liquid (Sigma-Aldrich Perfluorohexane $\mathrm{C}_{6} \mathrm{~F}_{14}$ ) during the measurement. A small fraction of the monochromatic pump light was split off as intensity reference and measured by lock-in detection (Stanford Research Systems SR830 lock-in amplifier) using a pyro-electric detector (Newport DET-L-PYC5-R-P). Thin film samples for PDS were prepared on cleaned quartz substrates by spin-coating. A stabilized cw-laser (Thorlabs HR S015 HeNe 633nm) was used as probe beam source focused closely to the sample surface. The deviation of the probe beam was detected by a silicon quadrant detector (Thorlabs PDP90A) using lock-in detection (Stanford Research Systems SR830). The entire setup was controlled by a home-built LabView-based data acquisition and device control code. The PDS spectra were set to absolute scale by matching the spectra with integrating sphere measurements on a Varian Cary 6000 spectrophotometer.

7) J-V characterization. Current-voltage characteristics were measured with a Keithley 2400 source measuring unit under simulated AM1.5 illumination (Sciencetech class A). Devices were tested under a continuous nitrogen flow. An aperture was used for measurement. The area of the used aperture is $0.049 \mathrm{~cm}^{2}$. The IV curves were scanned from $-0.70 \mathrm{~V}$ to $+0.1 \mathrm{~V}$ at $0.02 \mathrm{~V}$ interval steps without wait time between voltage steps. The spectral mismatch was calibrated using a reference solar cell (Newport). 
8) EQE measurement. External-quantum-efficiency spectra were taken by subjecting the solar cells to chopped $(220 \mathrm{~Hz})$ monochromatic illumination (400W Xe lamp passing through a monochromator and appropriate cut-off filters). Newport 818-UV and Newport 838-IR photodetectors were used to calibrate the output power. The response of the cell was measured with a Lakeshore preamplifier feeding into a Stanford Research 830 lock-in amplifier at short-circuit conditions. For high dynamic range EQE measurement, the sensitivity of preamplifier is selected to $1 \mathrm{nA} / \mathrm{V}$ to ensure the resolution of EQE at nearinfrared region $(>1100 \mathrm{~nm})$.

\section{Reference}

(44) Ning, Z. et al. Graded doping for enhanced colloidal quantum dot photovoltaics. Adv. Mater. 25, 1719-1723 (2013).

(45) Kunneman, L. T. et al. Nature and decay pathways of photoexcited states in CdSe and CdSe/CdS nanoplatelets. Nano Lett. 14, $7039-7045$ (2014). 


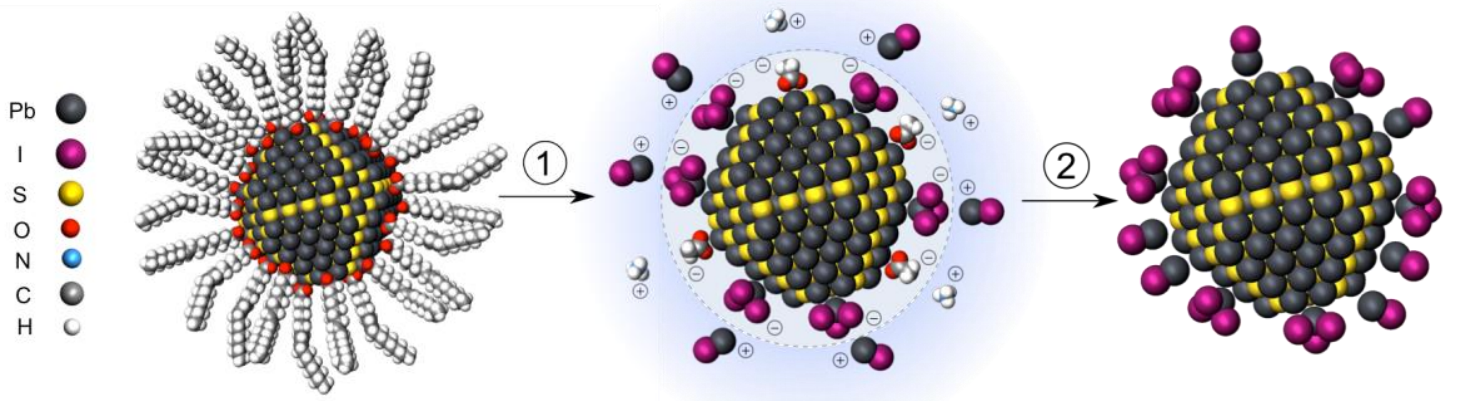

Figure 1. Solution-phase ligand exchange with metal halide precursors and ammonium acetate. Process 1 (ligand exchange): the bulky oleic acid ligands are replaced by the $\left[\mathrm{PbX}_{3}\right]^{-}$anions with the aid of ammonium protons. The CQD surface is stabilized by both $[\mathrm{PbX}]^{+}$and $\left[\mathrm{NH}_{4}\right]^{+}$. Process 2 (CQD precipitation): after ligand exchange, CQDs are precipitated via the addition of toluene, an anti-solvent, and are separated by centrifugation. $\mathrm{NH}_{4} \mathrm{Ac}$ is taken away by the mixed solvent and thus clean $\left[\mathrm{PbX}_{3}\right]^{-}$-capped CQD solids are left without any organic residues. 

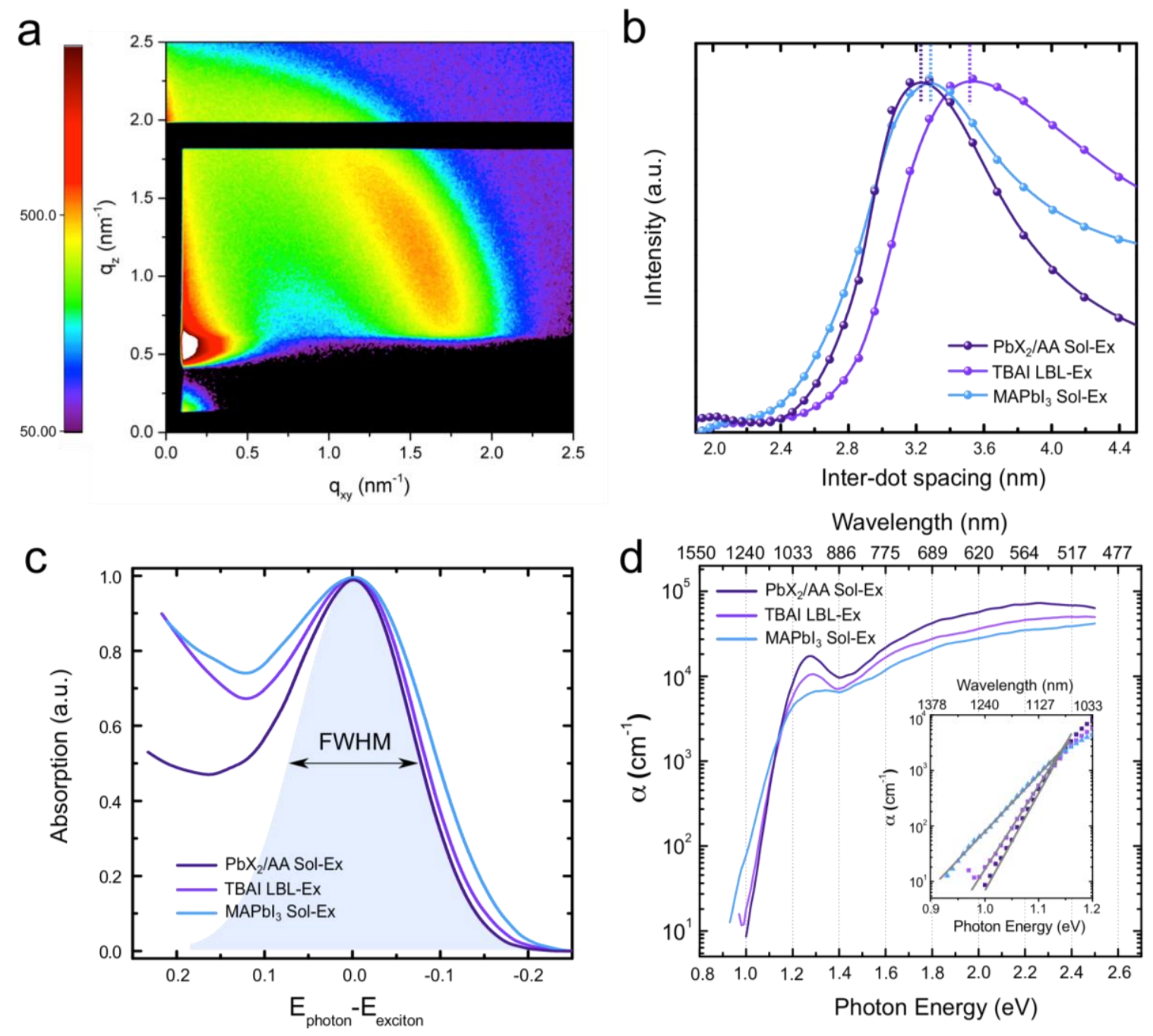

Figure 2. PbS CQDs exchanged by lead halide with aid of ammonium acetate suggests improved CQD packing density and sharper bandtail. (a) GISAXS 2D pattern of the $\mathrm{PbX}_{2} / \mathrm{AA}$ exchanged film. The color scale represents the log of the scattering intensity as recorded by the detector. Blue represents lower intensity and red represents higher intensity, with each spectrum normalized to show the full dynamic range of each data set. (b) Azimuthally integrated GISAXS intensities show the distribution of inter-dot spacing for different films; solution-exchanged films had smaller d-spacing, indicating denser CQD packing. (c) Normalized optical absorption of films after ligand exchange. The curves are fitted to a Gaussian distribution. The FWHM of the Gaussian fitting is used to display the monodispersity and packing homogeneity of the films. (d) Photothermal deflection spectroscopy (PDS) measurements for three different films. The Urbach energy calculated is $29 \mathrm{meV}$ for $\mathrm{PbX}_{2} / \mathrm{AA}$ exchanged film, $33 \mathrm{meV}$ for TBAI and $44 \mathrm{meV}$ for $\mathrm{MAPbI}_{3}$ exchanged one. 

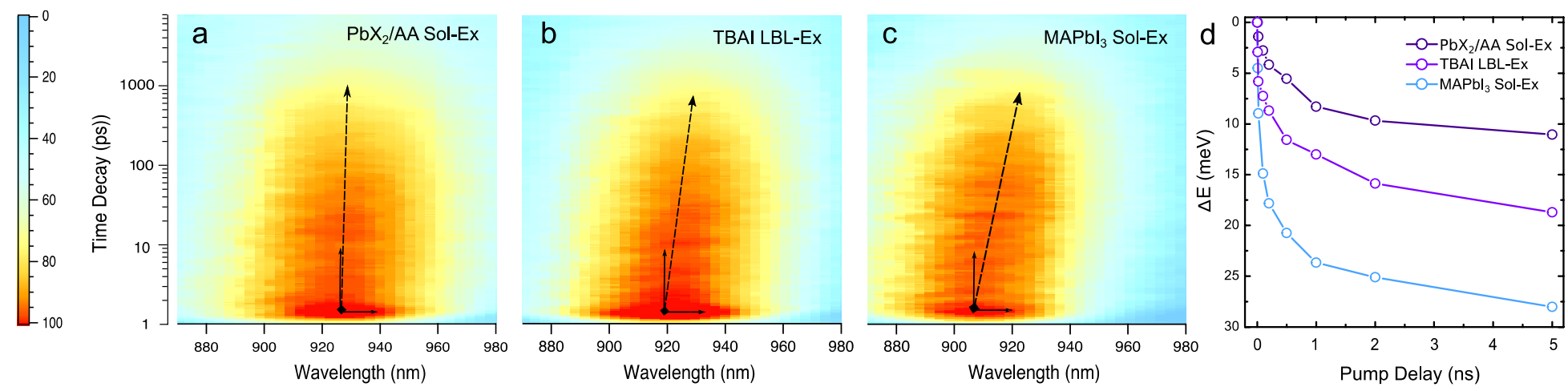

Figure 3. Energy funneling in the exchanged CQD films. Spectro-temporal transient absorption maps for (a) $\mathrm{PbX}_{2} / \mathrm{AA}$ solution exchanged films, (b) TBAI solid-state exchanged films, and (c) perovskite solution exchanged films. The samples are photoexcited at the low excitation density of $\left\langle\mathrm{N}_{\mathrm{abs}}\right\rangle=0.004$. A contour filter was applied to improve the signal-tonoise ratio. The peak positions of the transient bleach spectra red shift, to different extents, during recovery processes. (d) The shifts of peak position of transient bleach over the time for different exchanged films. The bleaching peak shifts around $11 \mathrm{meV}$ for $\mathrm{PbX}_{2} / \mathrm{AA}$ exchanged films, much less than $18 \mathrm{meV}$ for TBAI-exchange films and $28 \mathrm{meV}$ for $\mathrm{MAPbI}_{3}$-exchanged ones. This indicates less energy funneling in $\mathrm{PbX}_{2} / \mathrm{AA}$ exchanged films. 

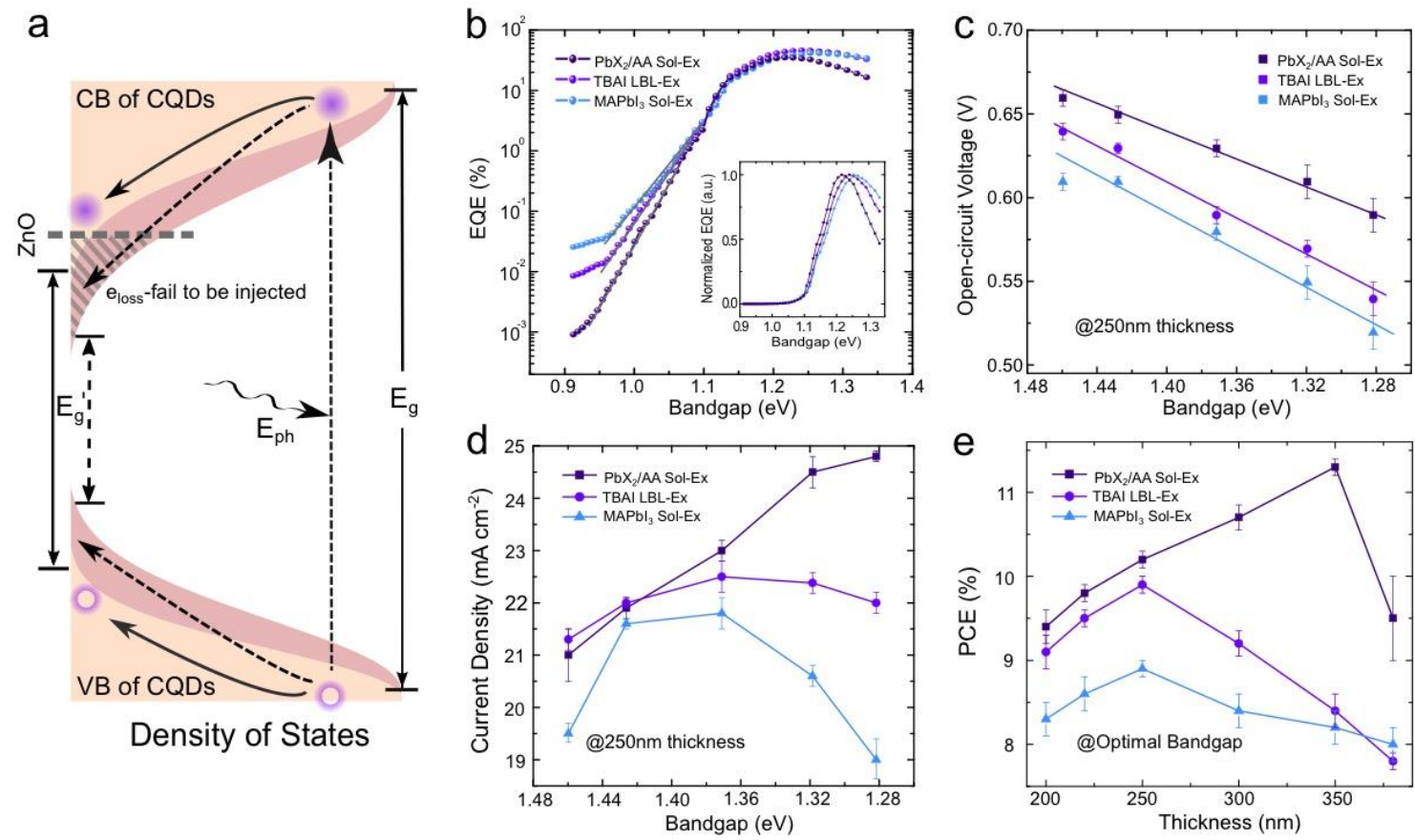

Figure 4. The effect of flat-energy-landscape on CQD solar cell performance. (a) A schematic of carrier thermalization in quantum dot films. Excitons are produced via solar irradiation, and then quickly thermalize to the bottom of density of states. The tail states filled with carriers introduce an effective bandgap $\left(\mathrm{E}_{\mathrm{g}}{ }^{\prime}\right)$ that limits the $\mathrm{V}_{\mathrm{oc}}$ of optoelectronic devices. Additionally, when the tail states are deeper than the conduction band of $\mathrm{ZnO}$, the charge injection from CQDs to $\mathrm{ZnO}$ is inhibited (shaded region), thus reducing the photocurrent. By reducing or eliminating the tail states, the electrons occupy higher energy levels in the conduction band and lower energy levels in the valence band, enabling a higher $\mathrm{V}_{\text {oc }}$ limit and more efficient carrier injection at the interface. (b) HDR EQE measurement to investigate the device-level carrier distribution over the energy range below the bandgap. (c) $V_{o c}$ and (d) $J_{s c}$ of 3 types of devices with different size of CQDs. (e) Device performances with different active layer thicknesses. Three types of devices are prepared at their optimal bandgap: $1.32 \mathrm{eV}$ for $\mathrm{PbX}_{2} / \mathrm{AA}$ exchanged film, and $1.43 \mathrm{eV}$ for both TBAI and $\mathrm{MAPbI}_{3}$ exchanged films. 

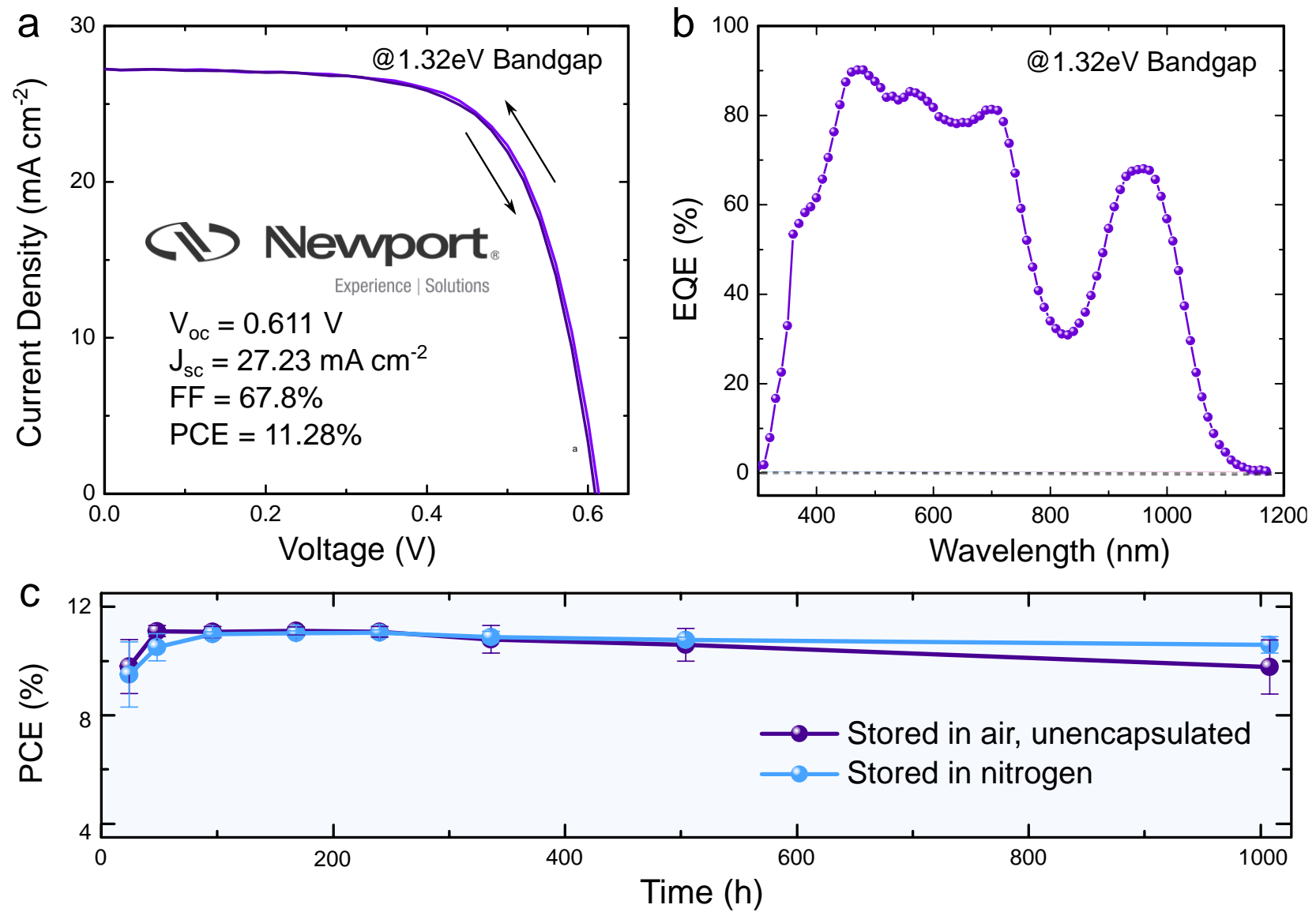

Figure 5. Certified solar cell performance. Performance of certified devices, (a) J-V curve, both forward and reverse scan, and (b) EQE. (c) Stability test for devices stored in air and nitrogen. 